\title{
Measuring The Psychological Attitudes of Non-Specialist Academic Staff of Al- Maarif University College Toward Practicing Sports
}

\author{
Othman Adham Ali \\ Department of Physical Education and Sport Science, Al-Maarif University College, Iraq \\ College of Physical Education, University of Anbar, Iraq \\ othmanadhamali@uoa.edu.iq
}

KEYWORDS: $\quad$ Psychological Attitudes, Sports Activity, Academic Staff, Psychology.

\begin{abstract}
:
Psychological attitudes are one of the most important topics in the field of psychology as one of the most important drivers of human behavior, as the psychological attitudes of academic employees have a great role in scientific development in all fields, especially in the sports field. Thus, psychological attitudes are the cornerstone in obtaining accurate scientific information about the composition of the measured phenomenon. As well as the importance of sports activities. Hence, the importance of the research emerges by recognizing the psychological attitudes of the academic staff of Al-Maarif University College for non-specialization towards the practice of sports activity. Which contributes to strengthening their attitudes towards the activity, modifying it, or changing it. The aim of the research is to identify the psychological attitudes of the academic staff of Al-Maarif University College for nonspecialists towards practicing sports activity. Then the researcher adopted the descriptive approach in the survey method for its relevance to the field research problem, and the research sample was chosen in a deliberate way from the total study population, where the number of study sample members reached (100) teaching, and after using the scale of the psychological attitude and obtaining answers, analyzing and discussing them, the researcher concluded. That the degrees of attitudes of academic staff of the departments of Al-Maarif University College according to the attitude scale indicate that their attitudes are positive towards the practice of sports activities. The researcher also recommended increasing social awareness of the benefit of exercising on the physical and psychological levels through holding seminars, using the media, and issuing books that explain the benefit of exercising.
\end{abstract}


قياس الاتجاهات النفسية لتدريسي كلية المعارف الجامعة لغير الاختصاص نخو ممارسة النشاط الرياضي

\author{
عثمان ادهام علي \\ قسم التربية البدنية وعلوم الرياضة، كلية المعارف الجامعة، العراق

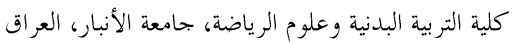 \\ othmanadhamali@uoa.edu.iq
}

$$
\text { الكلمات المفتاحية | الاتجاهات النفسية، النشاط الرياضي، تدريسين، علم النفس. }
$$

Crossref doi https://doi.org/10.51345/.v33i1.441.g260

\title{
ملخص البحث:
}

تعد الاتجاهات النفسية من اهم الموضوعات في مجال علم النفس باعتبارها من اهم محركات السلوك الإنساني، اذ ان لإتلاهاهات

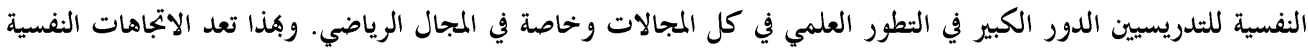

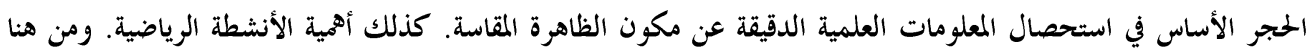

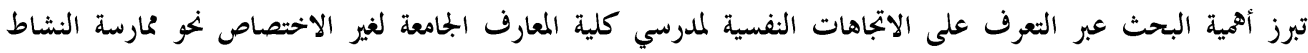

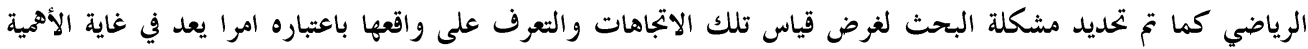

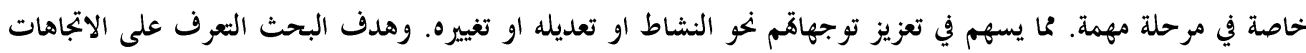

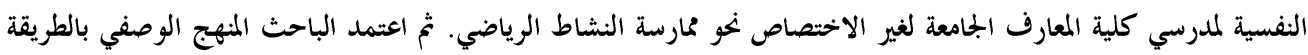

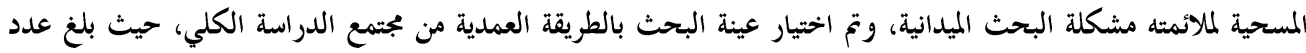

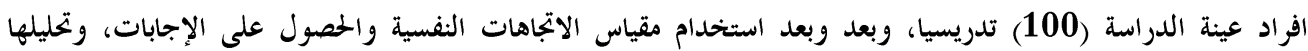

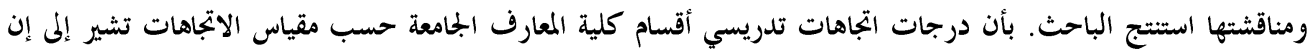

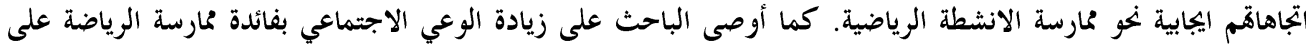

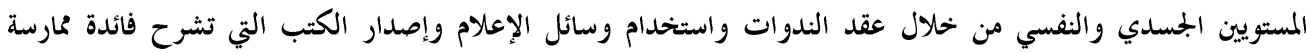

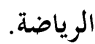

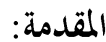

تعد الاتجاهات النفسية في الوقت الحاضر من أهم الموضوعات في بحال علم النفس كأحد أهم محركات السلوك البشري. من خلال ميل الشخص إلى ظاهرة أو حدث معين يمكننا التبؤُ بسلو كه اللاحق بتحاه

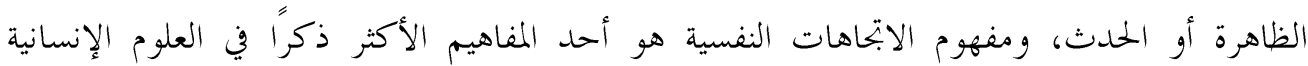
والاجتماعية لأنه طريقة منظمة التفكير والشعور، ويرتبط بردود الفعل على مواقف من حوله في البيئة. 
تتكون البيئة المحيطة من أفراد أو قضايا اجتماعية مختلفة، لذا فإن جميع الظواهر النفسية والاجتماعية

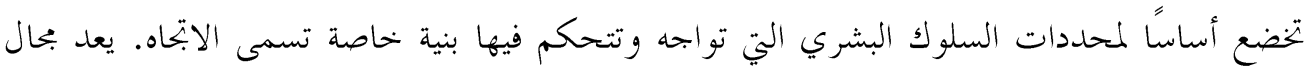

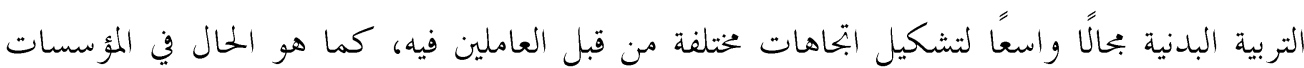
التعليمية. النجاح فيه يعتمد على تعاون وتضامن جميع القيادات التربوية من الأقسام أو الكو ادر التعليمية.

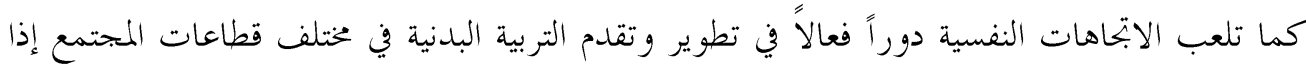

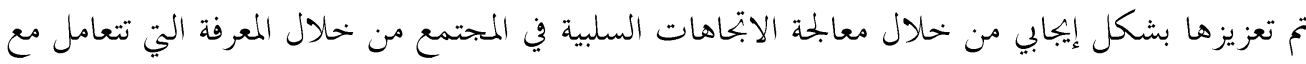
الجوانب المختلفة وتحديد قيمة وأهمية التربية البدنية في المجتمع ولفت الانتباه إلى حقيقة أن التربية البدنية

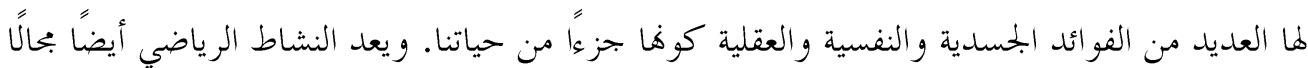

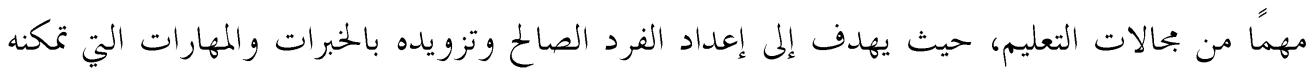

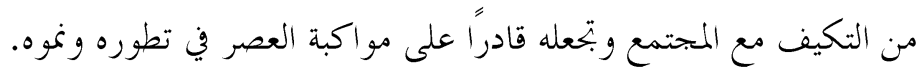
ومن هنا تأتي أهمية الدراسة من خلالال التعرف على الاتحاهات النفسية لمدرسي كلية المعارف الجحامعة في الأنبار لغير الاختصاص نهو منارسة النشاط الرياضي. كما أنه دافع لمدرسي التربية البدنية للقيام بواجباهم و استعدادهم للارتقاء وتحسين درس التربية البدنية والنشاط الرياضي وكذلك كسب دمب دعم المدرسين من

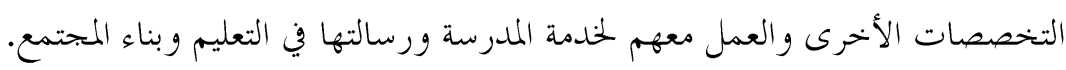

\section{مشكلة البحث:}

تتطلب الاتحاهات النفسية للمدرسين من تخصصات أخرى غير تخصص التربية البدنية البحث وتوضيح كافة الأبعاد التي تنطوي عليها، ومواجهة الاتحاهات السلبية بتحاه النشاط الرياضي الصفي واللامنهجي، وتطوير الحلول المناسبة لمم، وكسب دعم أعضاء هيئة التدريس.. و.ما أن الاتحاهات النفسية للمدرسين

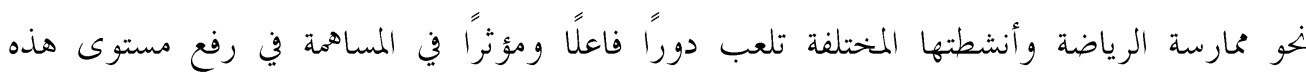

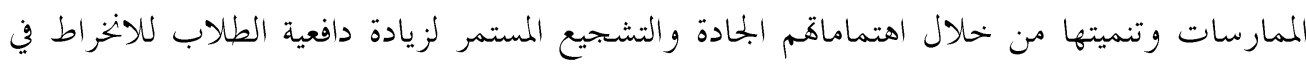

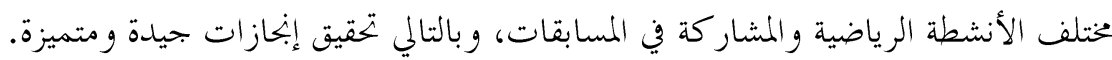

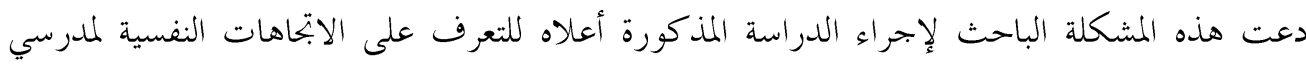
كلية المعارف الجمامعة غير المتخصصين بتحاه ممارسة النشاط الرياضي. 


\section{هدف البحث:}

التعرف على الاتجاهات النفسية لمدرسي كلية المعارف الجحامعة لغير الاختصاص نهو ممارسة النشاط الرياضي.

\section{مجالات البحث:}

المجال البشري: مدرسي كلية المعارف الجامعة لغير الاختصاص

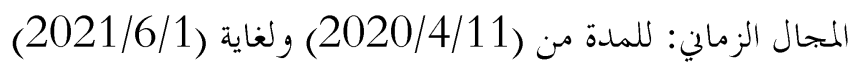
المجال المكاني: كلية المعارف الجامعة.

\section{تحديد المصطلحات:}

القياس: هو بحموعة مرتبة من المثيرات اعدت لتقيس بطريقة كمية او بطريقة كيفية بعض العمليات او السمات او الخصائص النفسية (1). الاتحاه النفسي: هو استعداد وجداني مكتسب ثابت نسبياً يمدده شعور الفرد وسلو كه ازاء موضوعات معينة او عدم تفضيلها (2).

النشاط الرياضي: هو ميدان من ميادين التربية عموما والتربية البدنية خصوصا، بحيث يعد عنصرا فعالا

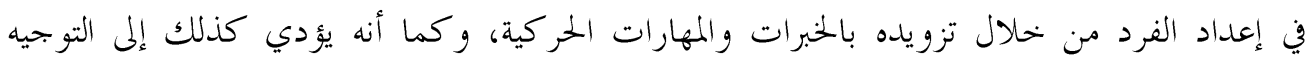

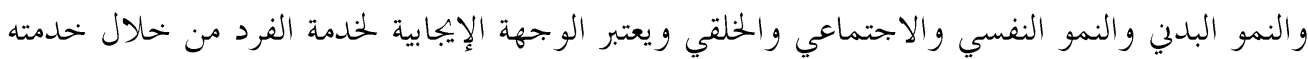

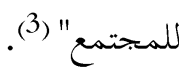

\section{منهج البحث واجر اءاته الميدانية}

\section{منهج البحث:}

استخدام الباحث المنهج الوصفي لملاءمته لطبيعة البحث.

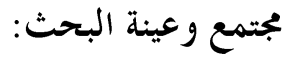

تم تحديد بحتمع البحث بالطريقة العمدية وهم مدرسي كلية المعارف الجامعة لغير الاختصاص وتكونت عينة البحث من (100) تدريسي توزعت على الأقسام التالية (المختبرات الطبية، واللغة العربية، واللغة

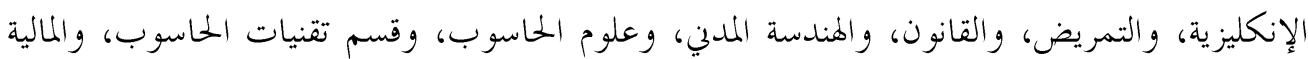




\section{الأدوات المستخدمة في البحث:}

استخدم الباحث مقياس الاتجاهات النفسية الذي وضعه عثمان ادهام لقياس الاتحاهات النفسية (4) لإدوات والذي يتكون من (60) فقرة ويستجيب كل فرد على كل عبارة في ضوء مقياس مدرج من خمسة أبعاد

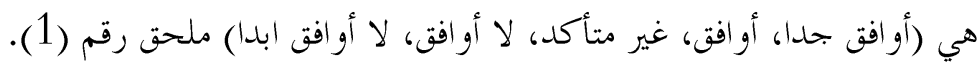

\section{الأسس العلمية للاختبارات:}

تم إيجاد المعاملات العلمية لاختبار الاتجاهات كما يلي. 1-الصدق: تم عرض المقياس على بحموعة من الخبراء والمختصين للتأكد من صلاحية تطبيقه على إفراد عينة البحث وقد أشارت آرائهم على صلاحية استخدام المقياس في قياس الاتحاهات. 2- الثبات: تم إيجاد الثبات عن طريق تطبيق المقياس ومن تم إعادة تطبيقه وذلك على عينه عينة عشو ائية قوامها (8) تدريسيين من كلية المعارف الجامعة، إذ تم توزيع الاستمارة على العينة الاستطلاعية يوم الأحد المصادف 2021/4/7 الساعة العاشرة صباحا وبعد سبع أيام تم إعادة توزيع الاستمارة على عينة

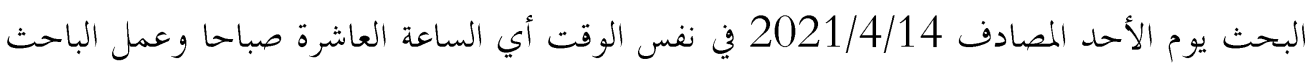

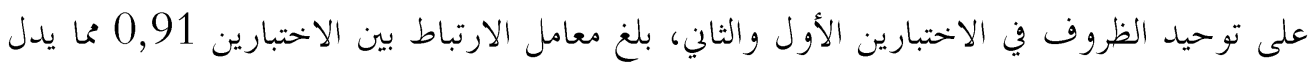
على إن المقياس على درجة عالية من الثبات.

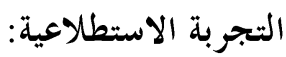

تم إجراء تجربة الرئيسية يوم السبت المصادف 2006/4/20 الساعة العاشرة صباحا للوقوف على

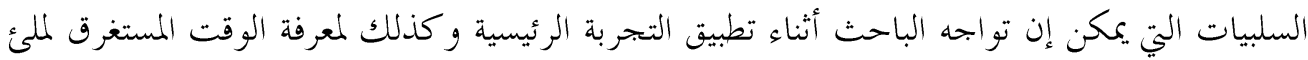

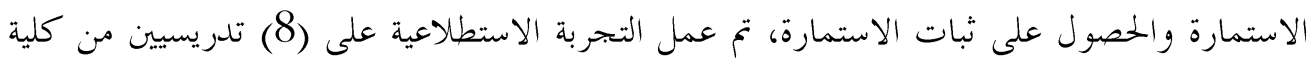
المعارف الجامعة.

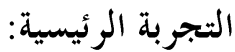

بعد الانتهاء من التأكد من صلاحية الاستمارة وإجراء التجربة الاستطلاعية تم توزيع الاستمارة يوم السبت المصادف 2021/4/27 على عينة البحث.

$$
\begin{aligned}
& \text { الوسائل الإحصائية (5): } \\
& \text { 1. الوسط الحسابي. }
\end{aligned}
$$




$$
\begin{aligned}
& \text { 2. الانخراف المعياري. } \\
& \text { 3. معامل الارتباط بيرسون. }
\end{aligned}
$$

\section{عرض النتائج وتحليلها ومناقشتها:}

\begin{tabular}{|c|c|c|c|c|}
\hline \multicolumn{5}{|c|}{ الاختصاص } \\
\hline الانحر اف المعياري & الوسط الحسابي & عدد التدريسيين & 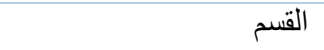 & $ت$ \\
\hline 8.3 & 180.23 & 14 & قسم القانون & 1 \\
\hline 9.95 & 182.71 & 12 & قسم المالية و الدصرفية & 2 \\
\hline 12.6 & 176.41 & 12 & قسم المختبرات الطبية & 3 \\
\hline 7.98 & 182.45 & 13 & قسم التمريض & 4 \\
\hline 8 & 185.89 & 11 & قسم اللغة العربية & 5 \\
\hline 8.43 & 188.53 & 13 & قسم اللغة الإنكليزية & 6 \\
\hline 9.21 & 182.78 & 13 & قسم علوم الحاسوب & 7 \\
\hline 8.27 & 182.23 & 12 & قسم هندسة تقنيات الحاسوب & 8 \\
\hline 9.09 & 182.56 & 100 & عينة البحث الكلية & 9 \\
\hline
\end{tabular}

الجدول (1) ييين عدد إفراد العينة والوسط الحسابي والانخر اف المعياري لمقياس الاتجاهات لمدرسي كلية المعارف الجامعة لغير

من الجحدول أعلاه كانت نتائج مقياس الاتحاه المطبق على عينة البحث كالاتي: كان الوسط الحسابي

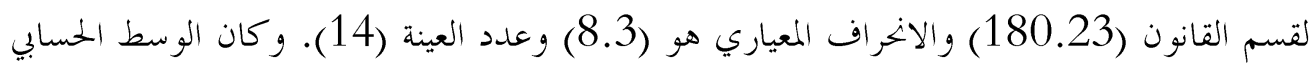

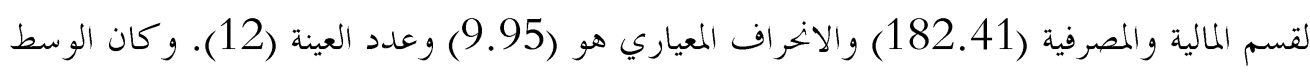
الحسابي لقسم المختبرات الطبية (176.41) والانحراف المعياري هو (12.6) وعدد العينة (12). وكان الوسط الحسابي لقسم التمريض (182.45) والانحراف المعياري هو (13.98) وعدد العينة

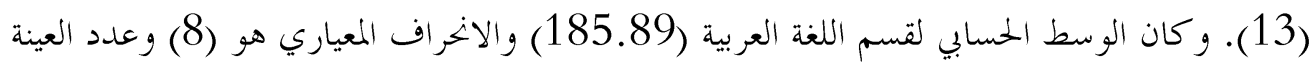

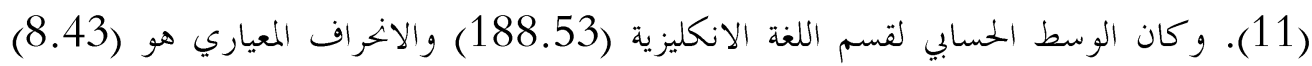

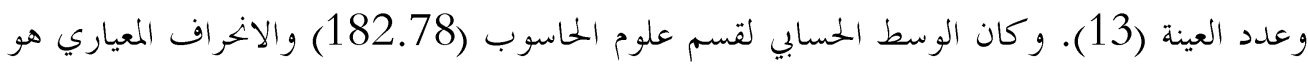
(9.21) وعدد العينة (13). و كان الوسط الحسابي لقسم هندسة تقنيات الحاسوب (182.56) والانحراف المعياري هو (8.27) وعدد العينة (12). من الجدول وعلدد أعلاه نلاحظ أن نتائج المقياس تشير

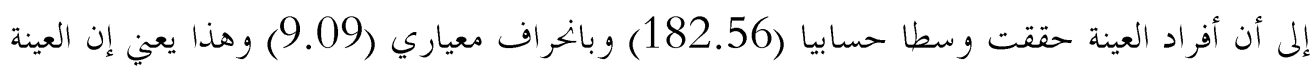


بشكل عام كانت بالابجاهات الايجابية نهو الانشطة الرياضية. حيث تعتبر العينة من الطبقة المثقفة و الواعية في المجتمع ، والتي تنظر إلى الرياضة على أها ذات تأثير إيهابي على الجسم والصحة من جهة، وعلى الروح والعلاقات الاجتماعية واحترام الذات والتعاون والحيوية تنمية احترام الذات واحترام الآخرين من ناحية أخرى.

من الجحدول نلاحظ أن قسم اللغة الإبحليزية حقق أعلى ابجاه إيجابي نهو الأنشطة الرياضية، ويعزو الباحث ذلك إلى حقيقة أن القسمين يتشار كان في مبنى واحد مما يستدعي تأثير مدرسي قسم اللغة الإبحليزية بسبب ذلك. تواصلهم الدائم مع مدرسي قسم التربية البدنية وعلوم الرياضة ، و كذلك تكوين صداقة بين المدرسين محا يزيد من فهمهم للرياضة والشعور بقضاء المرح والوقت المفيد. أما القسم الأقل من الابحاه نخو الأنشطة الرياضية فقد حققه قسم المختبرات الطبية، ويعزو الباحث ذلك إلى حقيقة أن مبنى

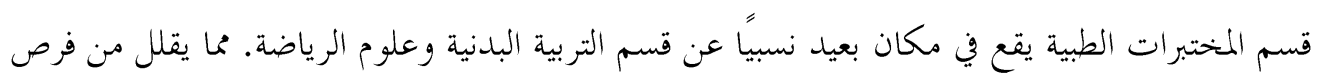
التأثر بالتمارين الرياضية أو التواصل مع قسم التربية البدنية وعلوم الرياضة.

\section{الاستنتاجات و التوصيات}

الاستنتاجات:

1- درجات ابجاهات تدريسي أقسام كلية المعارف الجامعة حسب مقياس الابحاهات تشير إلى إن ابتحاهاهم ايجابية نغو محارسة الانشطة الرياضية.

2- قسم اللغة الإنكليزية حقق أعلى ابتحاه نغو محارسة الانشطة الرياضية 3- قسم المختبرات الطبية حقق اقل ابتاه نخو ممارسة الانشطة الرياضية 4- إن الانشطة الرياضية في نظر التدريسيين الذين لا يمارسون النشاط الرياضي ذات فائدة صحية وتربوية.

\section{التوصيات:}

1- زيادة الوعي الاجتماعي بفائدة ممارسة الرياضة على المستو يين الجسدي والنفسي من خلال عقد الندوات واستخدام و سائل الإعلام وإصدار الكتب التي تشرح فائدة ممارسة الرياضة. 2- توعية مدرسي أقسام كلية المعرفة بالجامعة بأهمية الرياضة وإتاحة الفرصة فم لممارسة النشاط الرياضي. 3- إجراء أبحاث محاثلة لتدريسيين الكليات الأخرى وشريكة مختلفة من المجتمع. 


$$
\begin{aligned}
& \text { (1) سامي محمد ملحم: القياس والتقويم في التربية وعلم النفس، ط2، عمان، دار المسيرة، 2002، ص30. }
\end{aligned}
$$

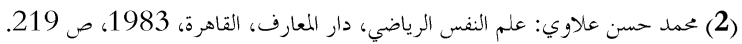

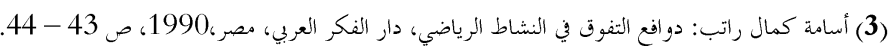

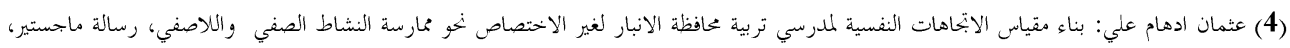
جامعة الانبار، كلية التربية البدنية وعلوم الرياضة، 2021.

(5) صلاح الدين محمود علام: القياس والتقويم التربوي والنفسي، أساسياته، تطبيقاته، توجيهاته المعاصرة، ط1، القاهرة، دار الفكر العربي، 2000، ص156.

\section{ملحق رقم (1)}

مقياس الاتجاهات النفسية لمدرسي تربية محافظة الانبار لغير الاختصاص نغو ممارسة النشاط الصفي واللاصفي ملي

\begin{tabular}{|c|c|c|c|c|c|c|}
\hline 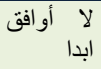 & 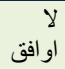 & غنأكد & 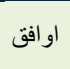 & 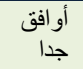 & 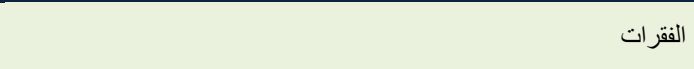 & $ت$ \\
\hline & & & & & ممارسة الانشطة الرياضية تكسبني عادات وفو ائد صحية & .1 \\
\hline & & & & & ارى ان اكتساب الفرد الصحة العامة لا يعني اكتساب اللياقة البدنية & .2 \\
\hline & & & & & ممارسة الأنشطة الرياضية تتيح لي فرصة الاتصال بالمجتمع & .3 \\
\hline & & & & & أجد صعوبة في التركيز الذهني في عمل معين بعد ممارسة النشاط الرياضي & .4 \\
\hline & & & & & ممارسة الرياضة تساعد في التعرف إلى المصطلحات و المفاهيم الرياضية & .5 \\
\hline & & & & & أخثى الاصابة بالعاهات البدنية جر اء ممارسة النشاط البدني & .6 \\
\hline & & & & & ارى ان عدم ممارسة الانشطة الرياضية تؤدي الى زيادة في وزن الفرد & .7 \\
\hline & & & & & للرياضيين مكانة اجتماعية جيدة في المجتمع & .8 \\
\hline & & & & & من الضغوط النفمبة الأنشطة الرياضية لأنها من أكثر الطرق جدوى في التخلص & .9 \\
\hline & & & & & الرياضية عن الحديث في المجال الرياضي لافتقاري للمعلومات والمعارف & 10 \\
\hline & & & & & أرى ان ممارسة الانشطة الرياضية بطريقة عشو ائية تؤدي الى الإصابات & 11 \\
\hline & & & & & أفضل ممارسة الانشطة الرياضية التي تكسبني اللياقة البدنية & 12 \\
\hline & & & & & ارى ان الألعاب الجماعية أكثر متعة من الألعاب الفردية & 13 \\
\hline & & & & & اشعر بالارتباك ولو قليلا عند مزاولتي للنشاط الرياضي & 14 \\
\hline & & & & & اود الحضور في المحاضر ات الني تعالج واقع الأنشطة الرياضية & 15 \\
\hline
\end{tabular}




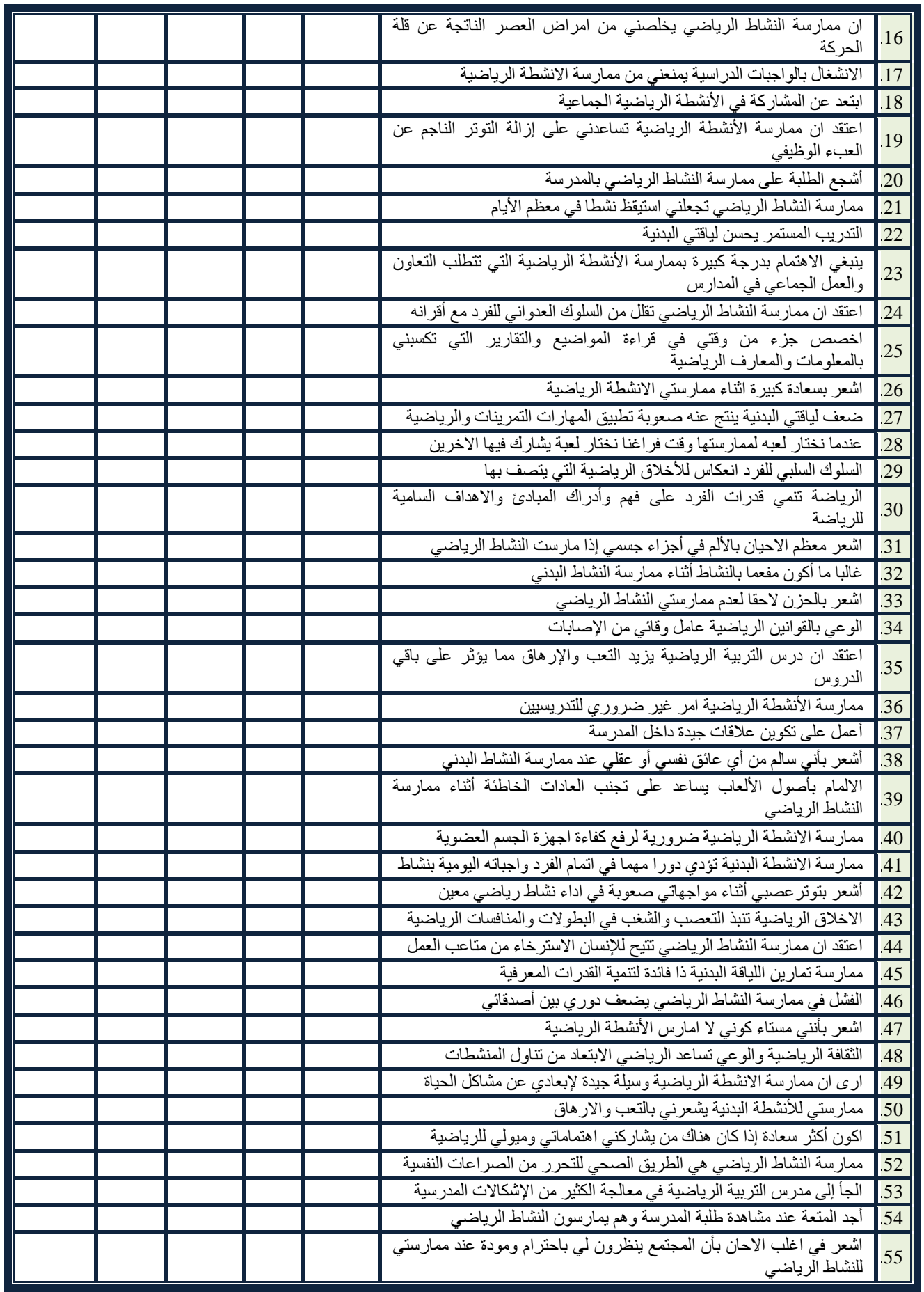




\begin{tabular}{|c|c|c|c|c|c|c|}
\hline & & & & & ممارسة الرياضة تساعدني على الاسترخاء و التخلص من الأرق و النوم بهلوء & 56 \\
\hline & & & & & أسنطع الإجابة عنه والاحر اج عند كل سؤ ال يخص الانشطة الرياضية يوجه لي ولم & .57 \\
\hline & & & & & ممارسة الأنشطة الرياضية تساعدني على تنمية الثعور بالمتعة و السعادة & 58 \\
\hline & & & & & الاجنماعية بينهة أنشاط الرياضي واقامة المسابقات بين الطلاب يعزز العلاقات & .59 \\
\hline & & & & & ممارسة الأنشطة الرياضية يسهم في بث الاتجاهات التربوية و التحلي بالخلق & 60 \\
\hline
\end{tabular}

